\title{
Discourse-Based Approach to Involvement of Background Knowledge for Question Answering
}

\author{
Boris Galitsky $^{1}$ and Dmitry Ilvovsky ${ }^{2}$ \\ ${ }^{1}$ Oracle Inc. Redwood Shores CA \\ ${ }^{2}$ National Research University Higher School of Economics \\ boris.galitsky@oracle.com; dilvovsky@hse.ru
}

\begin{abstract}
We introduce a concept of a virtual discourse tree to improve question answering $(\mathrm{Q} / \mathrm{A})$ recall for complex, multisentence questions. Augmenting the discourse tree of an answer with tree fragments obtained from text corpora playing the role of ontology, we obtain on the fly a canonical discourse representation of this answer that is independent of the thought structure of a given author. This mechanism is critical for finding an answer that is not only relevant in terms of questions entities but also in terms of interrelations between these entities in an answer and its style. We evaluate the Q/A system enabled with virtual discourse trees and observe a substantial increase of performance answering complex questions such as Yahoo! Answers and www.2carpros.com.
\end{abstract}

\section{Introduction}

In spite of the great success of search technologies, the problem of leveraging background knowledge is still on the agenda of search engineering, for both conventional and learning-based systems. Background knowledge ontologies are difficult and expensive to build, and knowledge graphs - based approaches usually have a limited expressiveness and coverage. In this study we explore how a discourse analysis (which is domainindependent) can substitute certain features of ontology-based search. There are few popular discourse theories describing how Discourse Trees (DT) can be constructed from the text. In our work we used Rhetorical Structure Theory (RST, Mann and Thompson, 1988).

Ontologies are in great demand for answering complex, multi-sentence questions with a precise answer in such domain as finance, legal, and health. In the educational domain this type of questions is referred to as convergent: answers to these types of questions are usually within a very limited range of acceptable accuracy. These may be at several different levels of cognition including comprehension, application, analysis, or ones where the answerer makes inferences or conjectures based on material read, presented or known. Answering convergent questions is an underexplored $\mathrm{Q} / \mathrm{A}$ domain that can leverage discourse analysis (Kuyten et al, 2015).

Discourse trees have became a standard for representing how thoughts are organized in text, in particular in a paragraph of text, such as an answer. Discourse-level analysis has been shown to assist in a number of NLP tasks where learning linguistic structures is essential (Louis et al., 2010; Lioma et al., 2012). DTs outline the relationship in between entities being introduced by an author. Obviously, there are multiple ways the same entities and their attributes are introduced, and not all rhetoric relations that hold between these entities occur in a DT for a given paragraph.

When DTs are used to coordinate questions and answers, we would want to obtain an "ideal" DT for an answer, where all rhetoric relations between involved entities occur. To do that, we need to augment an actual (available) DT of answer instance with a certain rhetorical relations which are missing in the given answer instance but can be mined from text corpora or from the web. Hence to verify that an answer $A$ is good for a given question $Q$, we first verify that their DTs $(D T$ $A$ and $D T-Q$ ) agree and after that we usually need to augment the $D T-A$ with fragments of other DTs to make sure all entities in $Q$ are 
communicated (addressed) in augmented $D T$ A.

Hence instead of relying on an ontology that would have definitions of entities which are missing in a candidate answer we mine for the rhetorical relations between these entities online. This procedure allows us to avoid an offline building of bulky and costly ontologies. At the same time, the proposed approach can be implemented on top of a conventional search engine.

The paper structure is as follows. In Section 2 we compare the related work with our proposal. In Section 3 we introduce the concept of a virtual discourse tree and present a number of examples illustrating how they can be used and constructed. In Section 4 we propose $\mathrm{Q} / \mathrm{A}$ filtering algorithm which is the core part of our approach. In Section 5 we describe and discuss evaluation for the question answering task on a few datasets that were compiled for this research.

\section{Related Work}

\subsection{Discourse and IR}

Typically, every part in most coherent text has some plausible reason for its presence, some function that it performs to the overall semantics of the text. Rhetorical relations, e.g. contrast, cause, explanation, describe how the parts of a text are linked to each other. Rhetorical relations indicate the different ways in which the parts of a text are linked to each other to form a coherent whole.

Marir and Haouam (2004) introduced a thematic relationship between parts of text using RST based on cue phrases to determine the set of rhetorical relations. Once these structures are determined, they are put in an index, which can then be searched not only by keywords, as traditional information retrieval systems do, but also by rhetorical relations.

It was observed (Teufel and Moens, 2002) that different rhetorical relations perform differently across evaluation measures and query sets. The four rhetorical relations that improve performance over the baseline consistently for all evaluation measures and query sets are: background, cause-result, condition and topic-comment. Topic-comment is one of the overall best-performing rhetorical relations, which in simple terms means that boosting the weight of the topical part of a document improves its estimation of relevance. Regretfully these relations are relatively rare.

Sun and Chai (2007) investigated the role of discourse processing and its implication on query expansion for a sequence of questions in scenario-based context Q/A. They consider a sequence of questions as a mini discourse. An empirical examination of three discourse theoretic models indicates that their discoursebased approach can significantly improve $\mathrm{Q} / \mathrm{A}$ performance over a baseline of plain reference resolution.

In a different task (Wang et al, 2010) authors parse Web user forum threads to determine the discourse dependencies between posts in order to improve information access over Web forum archives.

Suwandaratna and Perera (2010) present a re-ranking approach for Web search that uses discourse structure. They report a heuristic algorithm for refining search results based on their rhetorical relations. Their implementation and evaluation is partly based on a series of adhoc choices, making it hard to compare with other approaches. They report a positive userbased evaluation of their system for ten test cases.

Since rhetoric parsers for English (Joty et al., 2013, Surdeanu, 2015) have become more available and accurate, their application in search engine indexing is becoming more feasible. Precision and recall of search systems ignore discourse level information and users do not find products, services and information they need. It was shown that discourse features are valuable for passage re-ranking (Jansen et al., 2014). DTs have been also found to assist in answer indexing to make search more relevant: query keyword should occur in nucleus rather than a satellite of a rhetoric relation (Galitsky et al., 2015). In this study we go beyond leveraging discourse features and construct DTs from actual candidate answers and also virtual DTs for necessary background knowledge.

\subsection{Discourse Analysis and Entities}

At any point in the discourse, some entities are considered more salient than others (occurring in nucleus parts of DTs), and consequently are expected to exhibit different properties. In Centering Theory (Poesio et al., 2004), entity 
importance determines how they are realized in an utterance, including pronominalized relation between them.

Barzilay and Lapata (2008) automatically abstracts a text into a set of entity transition sequences and records distributional, syntactic, and referential information about discourse entities. The authors formulated the coherence assessment as a learning task and show that their entity-based representation is well-suited for ranking-based generation and text classification tasks.

Nguyen and Joty (2017) presented a local coherence model based on a convolutional neural network that operates over the distributed representation of entity transitions in the grid representation of a text, can model sufficiently long entity transitions and can incorporate entity-specific features without losing generalization power.

Kuyten et al., (2015) developed a search engine that leverages the discourse structure in documents to overcome the limitations associated with the bag-of-words document representations in information retrieval. This system does not address the problem of rhetoric coordination between $Q$ and $A$, but given a $Q$, this search engine can retrieve both relevant $\mathrm{A}$ and individual statements from $\mathrm{A}$ that describe some rhetorical relations to the query.

Our approach is to discover ontological relations between entities on the fly, finding document fragments where a rhetorical relation links these entities. Once all such text fragments are found, we add the respective DT fragments as virtual DTs to our main answer DT.

\section{Answering Questions via Discourse Trees}

\subsection{Virtual Discourse Tree}

The baseline requirement for an $A$ to be relevant to $Q$ is that entities $(E)$ of $A$ cover the entities of $Q$ :

$$
E-Q \subseteq E-A
$$

Naturally, some $E-A$ (entities in an answer) are not explicitly mentioned in $Q$ but are needed to provide a recommendation yielded by $Q$ (recipe-type $A)$.
The next step for an $A$ to be good for $Q$ is to follow the logical flow of $Q$. Since it is hard to establish relations between entities $E$, which are domain dependent, we try to approximate these relations by using logical flow of $Q$ and $A$, expressible in domain-independent terms, such as rhetorical relation. Hence we require a certain correspondence between DT-Q and DT-A, considering additional labels for DT nodes by entities (we denote such DT as EDT):

$$
E D T-Q \sim E D T-A .
$$

However a common case is that some entities $E$ are not explicitly mentioned in $Q$ but instead are assumed. Moreover, some entities in $A$ used to answer $Q$ do not occur in $A$ but instead are substituted by more specific or general entities do. How would we know that these more specific entities are indeed addressing issues from $Q$ ? We need some external, additional source which we call virtual EDT-A to establish these relationships.

This source contains the information on inter-relationships between $\mathrm{E}$ which is omitted in $Q$ and/or $A$ but is assumed to be known by the interlocutor. For an automated Q/A system, we want to obtain this knowledge at the discourse level:

$E D T-Q \sim E D T-A+$ virtual EDT-A.

\subsection{Discourse Trees for Answer and Question}

We start with a simple example:

Q: What is an advantage of electric car?

A: No need for gas.

How can search engine figure out that $A$ is a good one for $Q$ ? We have an abstract generalsense entity advantage and a regular noun entity car. We need to link explicit entities in $A$ $\{$ need, gas $\}$. Fragments of a possible virtual EDT-A are shown below:

Q: [When driving the cruise control][the engine will turn off][when I want to accelerate ,][although the check engine light was off.] [I have turned on the ignition][and listen for the engine pump running][to see][if it is building up vacuum .] [Could there be a problem with the brake sensor under the dash ?] [Looks like there could be a little play in the plug.] 


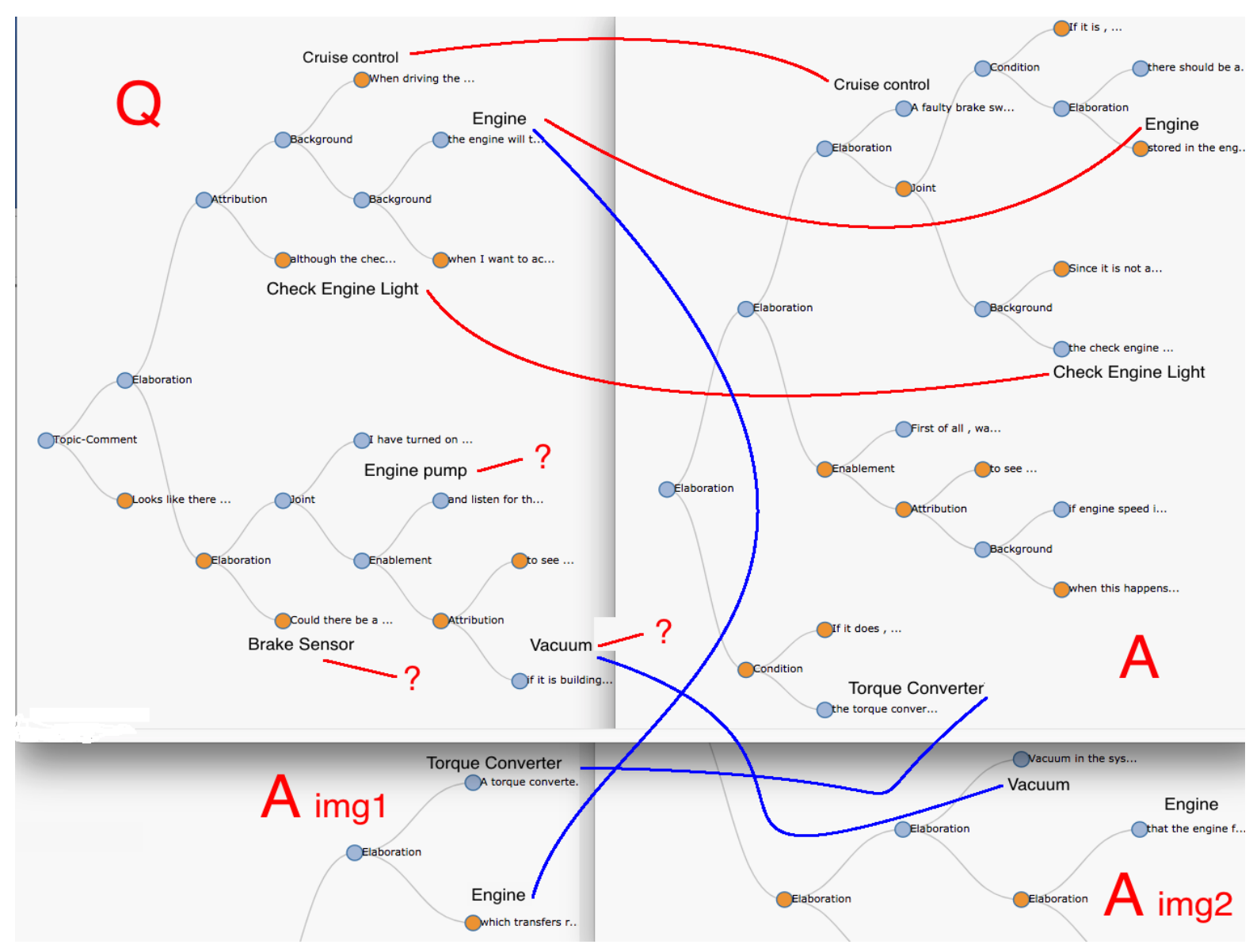

Figure 1: DTs of $Q, A$ and imaginary $D T-A_{i m g 1}$ and $D T-A_{i m g 2}$

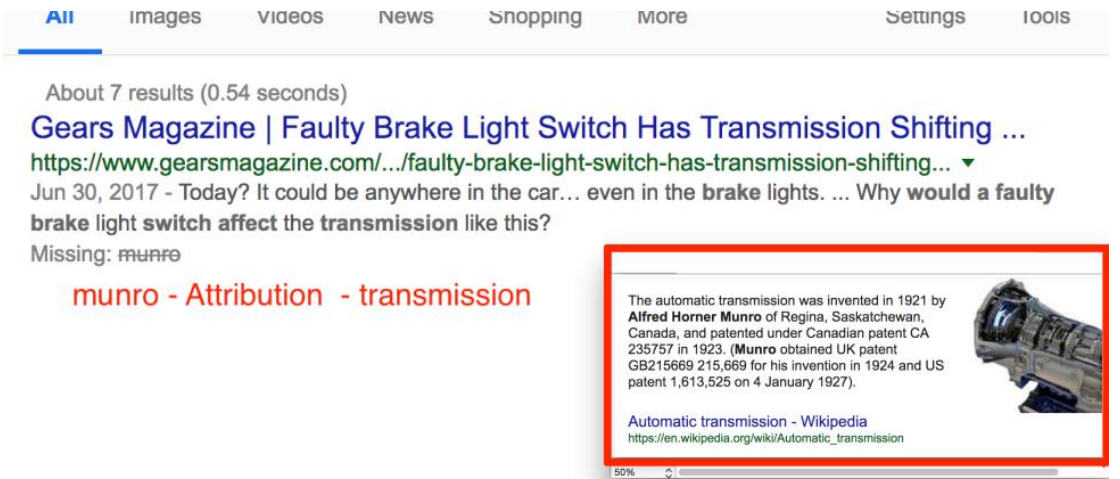

Figure 2: How Virtual DTs would enable Google search to explain missing keywords 
A: [A faulty brake switch can effect the cruise control .] [If it is,][there should be a code][stored in the engine control module.] [Since it is not an emissions fault,][the check engine light will not illuminate.] [First of all, watch the tachometer][to see][if engine speed increases 200 rpm][when this happens.] [If it does ,][the torque converter is unlocking transmission .]

We do not need to know the details concerning how this Enablement occurs, we just need evidence that these rhetorical links exist. We could have used semantic linked between entities but for that we would need a domainspecific ontology.

Let us explain how a match between a $Q$ and an $A$ is facilitated by DTs (Fig. 1). $A$ explains a situation and also offer some interpretation, as well as recommends a certain course of action. $A$ introduces extra entities which are not in $Q$, and needs to involve background knowledge to communicate how they are related to $E-Q$. We do it by setting a correspondence between $E-Q$ and $E-A$, shown by the horizontal curly (red) arcs.

Notice that some entities $E_{0}$ in $Q$ are unaddressed: they are not mentioned in $A$. $E_{0^{-}} Q$ includes \{Engine pump, Brake sensor and Vacuum $\}$. It means that either $A$ is not fully relevant to $Q$ omitting some of its entities $E_{0}$ or it uses some other entities instead. Are $E_{0^{-}} Q$ ignored in $A$ ? To verify the latter possibility, we need to apply some form of background knowledge finding entities $E_{\text {img }}$ which are linked to both $E_{0^{-}} Q$ and $E-A$.

It is unclear how $E-A=$ Torque Convertor is connected to $Q$. To verify this connection, we obtain a fragment of text from Wikipedia (or another source) about Torque Convertor, build $D T-A_{\text {imgl }}$ (shown on the left-bottom of Fig. 1) and observe that it is connected with Engine via rhetoric relation of elaboration. Hence we confirm that $E-A=$ Torque Convertor is indeed relevant for $Q$ (a vertical blue arc).

It is also unclear how E-Q pump is addressed in $Q$. We find a document on the web about Engine Pump and Vacuum and attempt to connect them to $E$ - $A$. It turns out that $D T-A_{\text {img } 2}$ connects Vacuum and Engine via elaboration.

Hence the combined $D T-A$ includes real $D T-A$ plus $D T-A_{\text {img } 1}$ and $D T-A_{\text {img } 2}$. Both real and virtual DTs are necessary to demonstrate that an answer is relevant by employing background knowledge in a domain independent manner: no offline ontology construction is required.

Search relevance is then measured as the inverse number of unaddressed $E_{0}-Q$ once $D T-A$ is augmented with virtual $D T-A_{\text {img }}$. This relevance is then added to a default one.

Fig. 2 shows an example how Virtual DT component would improve a web search. Currently, search engines show certain keywords they do not identify in a given search result. However, it is possible to indicate how these keywords are relevant to the search result by finding documents where these unidentified keywords are rhetorically connected with the ones occurring in the query. This feature would naturally improve the answer relevance on one hand and provide an "explainability" for the user

Algorithm 1 Filtering Algorithm

Input: Question

Parameter: Background knowledge $B$

Output: Most relevant Answer

1: Build $E D T-Q$.

2: Obtain $E-Q$

3: Form a query for $E-A$

4: Obtain a set of candidate answers $A s$

5: for each $A c$ in $A s$ do

6: $\quad$ Build discourse tree for the answer DT-Ac.

7: $\quad$ Establish mapping $E-Q \rightarrow E-A c$

8: $\quad$ Identify $E_{0}-Q$.

9: $\quad$ Form queries from $E_{0}-Q$ and $E_{0}-A c$ (entities which are not in $E_{0}-Q$ )

10: Obtain search results from $B$ for queries

11: Build imaginary DTs-Ac.

12: Calculate the score $=\left|E_{0}\right|$

\section{3:end for}

14:Select $A$ with the best score

\section{5:return $A$}

on how her keywords are addressed in the answer. In the default search, munro is missing. However, by trying to rhetorically connect munro with the entities in the question, the Virtual DT approach finds out that Munro is an 
inventor of automatic transmission. DT fragment is shown with rhetorical relation Attribution, as well as the Wikipedia source for virtual DT.

\section{Question Answering Approach}

\subsection{Question Answering Filtering Algorithm}

Given a Question, we outline an algorithm (Algorithm 1) that finds the most relevant Answer such that it has as much of $E$ - $Q$ addressed by $E-A$, having a source for virtual DTs (background knowledge) $B$.

Discourse trees are constructed automatically using state-of-the-art RST-parser (Surdeanu et.al, 2015).

\subsection{Learning on Q/A Pairs}

Besides this algorithm, we outline a machine learning approach to classify $\langle E D T-Q, E D T-A\rangle$ pair as correct or incorrect. The training set should include good Q/A pairs and bad Q/A pairs. Therefore a DT-kernel learning approach (SVM TK, Joty and Moschitti, 2014, Galitsky, 2017, 2018) is selected which applies SVM learning to a set of all sub-DTs of the DT for Q/A pair. Tree kernel family of approaches is not very sensitive to errors in parsing (syntactic and rhetoric) because erroneous sub-trees are mostly random and will unlikely be common among different elements of a training set.

Learning framework is available on our GitHub repository.

\section{Evaluation}

\subsection{Experiments on "Convergent" $\mathrm{Q} / \mathrm{A}$ Datasets}

Traditional Q/A datasets for factoid and nonfactoid questions, as well as SemEval and neural Q/A evaluations are not suitable since the questions are shorter and not as complicated to observe a potential contribution of discourse-level analysis. For our first evaluation, we formed two convergent $\mathrm{Q} / \mathrm{A}$ sets.

Yahoo! Answer ${ }^{1}$ set of question-answer pairs with broad topics. Out of the set of 140k user questions we selected 3300 of those, which included three to five sentences. Answers for most$$
1
$$

https://webscope.sandbox.yahoo.com/catalog.php?datatype=1
}

questions are fairly detailed so no filtering by sentence length was applied to the answers.

Car repair conversations (available online ${ }^{2}$ ) selected from www.2carpros.com including 9300 Q/A pairs of car problem descriptions vs recommendation on how to rectify them. These pairs were extracted from dialogues as first and second utterances so that a question is one to three sentences and answer is three to six sentences in length. Each dialogue is a comprehensive, cohesive sequence of questions and problem solving recommendations. Most recommendations include a set of conditions to check and actions to perform, not necessarily in the same terms as the problem was formulated. Therefore, traditional search engineering based on keyword statistics performs poorly on this dataset; both semantic and syntactic similarities between $\mathrm{Q}$ and $\mathrm{A}$ are low.

\begin{tabular}{|c|c|c|c|c|c|c|}
\hline \multirow{2}{*}{$\begin{array}{l}\text { Source } \\
\text { Search method }\end{array}$} & \multicolumn{3}{|c|}{ Yahoo! Answers } & \multicolumn{3}{|c|}{ Car Repair } \\
\hline & $\mathbf{P}$ & $\mathbf{R}$ & F1 & $\mathbf{P}$ & $\mathbf{R}$ & F1 \\
\hline $\begin{array}{l}\text { Baseline (Lucene } \\
\text { search engine) }\end{array}$ & 41.8 & 42.9 & 42.3 & 42.5 & 37.4 & 39.8 \\
\hline$|\mathrm{E}-\mathrm{Q} \cap \mathrm{E}-\mathrm{A}|$ & 53.0 & 57.8 & 55.3 & 54.6 & 49.3 & 51.8 \\
\hline 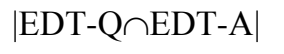 & 66.3 & 64.1 & 65.1 & 66.8 & 60.3 & 63.4 \\
\hline $\begin{array}{l}\mid \text { EDT-Q } \cap \text { EDT-A } \\
+ \text { EDT-A } \\
+ \text { imgi } \mid\end{array}$ & 76.3 & 78.1 & $\begin{array}{c}77.2 \pm \\
3.4\end{array}$ & 72.1 & 72.0 & $\begin{array}{l}72.0 \pm \\
3.6\end{array}$ \\
\hline $\begin{array}{l}\text { SVM TK for } \\
<\text { EDT-Q, EDT-A } \\
+ \text { EDT-A }_{\text {imgi }}>\end{array}$ & 83.5 & 82.1 & $\begin{array}{c}82.8 \pm \\
3.1\end{array}$ & 80. & 78.5 & $\begin{array}{c}79.6 \pm \\
4.1\end{array}$ \\
\hline
\end{tabular}

Human

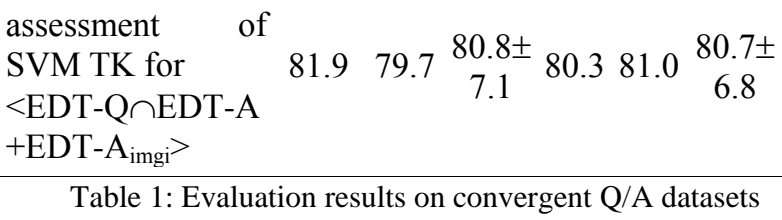

For each of these sets, we form the positive one from actual Q/A pairs and the negative one from $Q / A_{\text {similar-entities: }}$ E- $A_{\text {similar-entities }}$ has a strong overlap with E-A, although $A_{\text {similar-entities }}$ is not really correct, comprehensive and exact answer. Hence Q/A is reduced to a classification task measured via precision and recall of relating a Q/A pair into a class of correct pairs.

Top two rows in Table 1 show the baseline performance of $\mathrm{Q} / \mathrm{A}$ and demonstrate that in a complicated domain transition from keyword to

\footnotetext{
${ }^{2}$ https://github.com/bgalitsky/relevance-based-on-parsetrees/examples/CarRepairData_AnswerAnatomyDataset2.csv .zip.
} 
matched entities delivers more than $13 \%$ performance boost. For the baseline we used standard implementation of Lucene search engine based on matching keywords.

The bottom three rows show the Q/A quality when discourse analysis is applied. Assuring a rule-based correspondence between DT-A and DT-Q gives $13 \%$ increase over the baseline, and using virtual DT gives further $10 \%$. Finally, proceeding from rule-based to machine learned Q/A correspondence (SVM TK) gives the performance gain of about $7 \%$.

The difference between the best performing $S V M T K$ for $\left\langle E D T-Q \cap E D T-A+E D T-A_{\text {img } i}\right\rangle$ row and the above row is only the machine learning algorithm: representation is the same.

The bottom row shows the human evaluation of Q/A on a reduced dataset of 200 questions for each domain. We used human evaluation to make sure the way we form the training dataset reflects the $\mathrm{Q} / \mathrm{A}$ relevance as perceived by humans. This is important to confirm, in particular, that the negative dataset includes unsatisfactory answers. For a 1/3 fraction of this dataset we measured Krippendorff's alpha measure for the inter-annotator agreement (two annotators) which exceeds $80 \%$.

To summarize this experiment, the tree kernel learning of virtual discourse trees turned out to be a preferred approach. The contribution of virtual DTs might be insignificant for simpler, shorter, factoid questions when traditional measures of similarity between Q and A work well. However, we demonstrate that involvement of background knowledge via virtual DTs for complex convergent questions requiring entailment is significant.

\subsection{Experiments on a Standard Q/A Dataset}

We also compare the performance of virtual DT Q/A with neural extractive reading comprehension approaches (Table 2). We made this comparison on the why? and how-to? questions from SQuAD 2.0 (Rajpurkar et al., 2018), the dataset with unanswerable questions which look similar to answerable ones. In total 460 questions were selected.

Deep learning systems can often locate the correct answer to a question in a short text, but experience difficulties on questions for which the correct answer is not stated in the context. Rajpurkar et al. (2018) trained their system on
SQuAD and evaluated on the unseen questions. Whereas a deep learning system gets $86 \% \mathrm{~F} 1$ on SQuAD 1.1, it achieves only $66 \%$ on SQuAD 2.0 where some questions should not be answered.

\begin{tabular}{lcc}
\hline Approach & F1 & Reference \\
\cline { 1 - 1 } $\begin{array}{l}\text { BiDaf(Allen NLP) } \\
\text { (Gardner et al., 2017) }\end{array}$ & 64.8 & Our experiments \\
$\begin{array}{l}\text { DeepPavlov } \\
\text { (Burtsev et al., 2018) }\end{array}$ & 61.0 & Our experiments \\
$\begin{array}{l}\text { Microsoft Asia } \\
\text { (Hu et al., 2018) }\end{array}$ & 74.2 & $\begin{array}{c}\text { As reported by the } \\
\text { authors (full dataset) }\end{array}$
\end{tabular}

SVM TK for

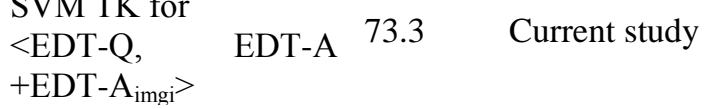

Table 2: Evaluation results on the SQuAD 2.0 dataset

We applied the model trained on Yahoo!Answers and Car Repair to our subset of questions from SQuAD 2.0. Because most unanswerable questions contain entities or entity types which do not occur in text, virtual DT is a good means to handle such cases. At the same time, by the nature of neural learning, it is hard to learn to refuse to answer. The best performance on SQuAD 2.0 for the totality of questions, including much simpler ones than our formed 460 questions dataset, is achieved by $(\mathrm{Hu}$ et al., 2018) and exceeds our model by less than $1 \%$.

The model of $\mathrm{Hu}$ et al. is specific to Wikipedia pages and the way questions are formulated, whereas our model learns once and for all which discourse structures is correlated with which forms of background knowledge. We believe this performance, achieved by training and testing on the same kind of $\mathrm{Q} / \mathrm{A}$ dataset is comparable with the results of the general model of the current study with the focus on convergent why/how to questions.

\section{Conclusions}

Answering questions in the domain of this study is a significantly more complex task than factoid Q/A such as Stanford Q/A dataset, where it is just necessary to involve one or two entities and their parameters. To answer a "how to solve a problem" question, one needs to maintain the logical flow connecting the entities in the questions. Since some entities from $Q$ are 
inevitably omitted, these would need to be restored from some background knowledge text about these omitted entities and the ones presented in $Q$. Moreover, a logical flow needs to complement that of the $Q$. The complexity of multi-sentence convergent questions, which was evaluated in, for example (Chen et al., 2017) is way below that one of a real user asking questions in the domains of the current study. Factoid, Wikipedia-targeted questions usually have fewer entities and simpler links between entities than the ones where virtual DT technique is necessary. At the same time, neural network based approach require a huge training set of $\mathrm{Q} / \mathrm{A}$ pairs which is rarely available in industrial, practical Q/A domains.

In spite of the great success of statistical and deep learning from a vast set of Q/A pairs, it is still hard to answer questions underrepresented in a training set. Most of the failures of learning approach occur when the user feels that the needed background knowledge is absent. The proposed technique does not require extensive training sets for all Q/A pairs which can be potentially encountered in real time. Instead, we consult necessary texts on demand in real time and avoid maintaining huge training sets on one hand and tackling extensive manually built ontologies on the other hand. Hence we propose a solution to one of the hardest and most sought after problem in AI of how to rely on background knowledge in industrial applications.

Domain-specific ontologies such as the ones related to mechanical problems with cars are very hard and costly to build. In this work we proposed a substitute via domain-independent discourse level analysis where we attempt to cover unaddressed parts of $D T-A$ on the fly, finding text fragments in a background knowledge corpus such as Wikipedia. Hence we can do without an ontology that would have to maintain relations between involved entities.

The proposed virtual DT feature of a $\mathrm{Q} / \mathrm{A}$ system delivers a substantial increase of performance answering complex convergent questions, where it is important to take into account all entities from a question. We observed that relying on rhetoric agreement between Q and A (matching their DTs) improves Q/A F1 by more than $10 \%$ compared to the relevance-only focused baseline. Moreover, employing virtual DTs gives us further $10 \%$ improvement.
Since we explored the complementarity relation between $D T-A$ and $D T-Q$ and proposed a way to identify virtual $D T-A$ on demand, the learning feature space is substantially reduced and learning from an available dataset of a limited size such as car repair becomes plausible.

\section{Acknowledgements}

Section 3 (algorithm to build a discourse representation for the domain knowledge) were written by Dmitry Ilvovsky supported by the Russian Science Foundation under grant 17-1101294 and performed at National Research University Higher School of Economics, Russia. Sections 4 and 5.2 (question answering algorithm, experimental investigations) were prepared within the framework of the HSE University Basic Research Program and funded by the Russian Academic Excellence Project '5100 '. The rest of the paper were written and performed at Oracle Corp.

\section{References}

A. Louis, A. Joshi, A. Nenkova. 2010. Discourse indicators for content selection in summarization. SIGDIAL, pp. 147-156.

Alexander Hogenboom, Flavius Frasincar, Franciska de Jong, and Uzay Kaymak. 2015. Using rhetorical structure in sentiment analysis. Communications of the ACM 58(7):69-77.

Boris Galitsky, D. Ilvovsky, and S. Kuznetsov. 2015. Rhetoric Map of an Answer to Compound Queries. ACL-2, 681-686.

Boris Galitsky, D. Ilvovsky, and S. Kuznetsov. 2018. Detecting logical argumentation in text via communicative discourse tree. JETAI. pp 637-663.

Boris Galitsky. 2017. Discovering Rhetorical Agreement between a Request and Response. Dialogue \& Discourse 8(2) 167-205.

Boris Galitsky. 2014. Learning parse structure of paragraphs and its applications in search. Engineering Applications of Artificial Intelligence. $32,160-84$

Chali, Y. Shafiq R. Joty, and Sadid A. Hasan. 2009. Complex question answering: unsupervised learning approaches and experiments. J. Artif. Int. Res. 35, 1 (May 2009), 1-47.

Chen D, A Fisch, J Weston, A Bordes. 2017. Reading Wikipedia to answer open-domain questions. https://arxiv.org/abs/1704.00051. 
Christina Lioma, Birger Larsen, and Wei Lu. 2012. Rhetorical relations for information retrieval. SIGIR, Portland, OR, pp. 931-940.

D. T. Nguyen and S. Joty. A Neural Local Coherence Model. 2017. ACL. pp. 1320-1330.

Gardner, M., Grus, J., Neumann, M., Tafjord, O., Dasigi, P., Liu, N.H., Peters, M., Schmitz, M., \& Zettlemoyer, L.S. A Deep Semantic Natural Language Processing Platform. arXiv:1803.07640.

Jansen, P., M. Surdeanu, and Clark P. 2014. Discourse Complements Lexical Semantics for Nonfactoid Answer Reranking. ACL.

Joty, Shafiq R and A. Moschitti. Discriminative Reranking of Discourse Parses Using Tree Kernels. EMNLP 2014.

Joty, Shafiq R, Giuseppe Carenini, Raymond $\mathrm{T} \mathrm{Ng}$, and Yashar Mehdad. 2013. Combining intra-and multi- sentential rhetorical parsing for documentlevel discourse analysis. In $A C L$ (1), pages 486496.

M. Burtsev, A. Seliverstov, R. Airapetyan, M. Arkhipov, D. Baymurzina, N. Bushkov, O. Gureenkova, T. Khakhulin, Y. Kuratov, D. Kuznetsov, A. Litinsky, V. Logacheva, A. Lymar, V. Malykh, M. Petrov, V. Polulyakh, L. Pugachev, A. Sorokin, M. Vikhreva, M. Zaynutdinov. 2018. DeepPavlov: Open-Source Library for Dialogue Systems. ACL-System Demonstrations, p. 122127. 2018.

M. Sun and J. Y. Chai. 2017. Discourse processing for context question answering based on linguistic knowledge. Know.-Based Syst., 20:511-526, August 2007.

Marir F. and K. Haouam. 2004. Rhetorical structure theory for content-based indexing and retrieval of Web documents, ITRE 2004, pp. 160-164.

Minghao Hu, Furu Wei, Yuxing Peng, Zhen Huang, Nan Yang, Dongsheng Li. 2018. Read + Verify: Machine Reading Comprehension with Unanswerable Questions. arXiv:1808.05759

P. Kuyten, D. Bollegala, B. Hollerit, H. Prendinger and K. Aizawa. 2015. A Discourse Search Engine Based on Rhetorical Structure Theory. Advances in Information Retrieva (ECIR). pp 80--91.

Poesio, M., R. Stevenson, B. Di Eugenio, and J. Hitzeman. 2004. Centering: A parametric theory and its instantiations. Computational Linguistics, 30(3):309-363.

Pranav Rajpurkar, Robin Jia, Percy Liang. Know What You Don't Know: Unanswerable Questions for SQuAD. arxiv.org/abs/1806.03822
Pranav Rajpurkar, Zhang, Jian; Lopyrev, Konstantin; Liang, Percy. 2016. SQuAD: 100,000+ Questions for Machine Comprehension of Text. in EMNLP 2016.

Regina Barzilay and Mirella Lapata. Modeling local coherence: An entity-based approach. Comput. Linguist. 34, 1 (March 2008), 1-34.

S. Teufel and M. Moens. Summarizing scientific articles: Experiments with relevance and rhetorical status. Computational Linguistics, 28(4):409-445. 2002.

Surdeanu, Mihai, Thomas Hicks, and Marco A. Valenzuela-Escarcega. 2015. Two Practical Rhetorical Structure Theory Parsers. NAACL HLT.

Suwandaratna, N. and U. Perera. 2010. Discourse marker based topic identification and search results refining. In Information and Automation for Sustainability (ICIAFs), 2010 5th International Conference on, pages 119-125.

Wang, W., Su, J., Tan, C.L. 2010. Kernel Based Discourse Rela-tion Recognition with Temporal Ordering Information. ACL.

William Mann and Sandra Thompson. 1988. Rhetorical Structure Theory: Toward a functional theory of text organization. Text 8(3):243-281.

Yangfeng Ji and Noah Smith. 2017. A Neural Discourse Structure for Text Categorization. ACL 2017. 